\section{Differential acquired resistance to anti-TNF therapy in RA patients}

Loss of effectiveness of traditional diseasemodifying antirheumatic drugs (DMARDs) is a common problem for patients with rheumatoid arthritis (RA), and the development of resistance to anti-tumor necrosis factor (TNF) therapy is starting to be recognized. A study by Finckh et al. has now shown that acquired resistance to anti-TNF therapies differs between agents.

The study included 1,198 patients with RA, treated with infliximab, etanercept or adalimumab, identified from The Swiss Clinical Quality Management of RA System. Data were collected between January 1998 and September 2004. Several outcomes indicated resistance to anti-TNF therapy: an increase in concomitant DMARD treatment, dose escalation of anti-TNF treatment, and anti-TNF discontinuation.

The results showed that infliximab was associated with a higher risk of requiring intensification of co-therapy with traditional DMARDs compared with etanercept or adalimumab, and that dose escalation often occurred in infliximab-treated patients, but not in patients treated with etanercept or adalimumab. Discontinuation rates were similar for all three anti-TNF agents, with a median duration of therapy of 3.21 years.

The authors comment that the dose escalation associated with infliximab could have resulted from initial dose adjustments, rather than from acquired infliximab resistance; however, both phenomena are likely to be involved. This study had some limitations, including a short follow-up of patients treated with adalimumab, because of its recent launch. The authors conclude that further research into the mechanisms of anti-TNF resistance is required.

Original article Finckh A et al. (2006) Evidence for differential acquired drug resistance to anti-tumour necrosis factor agents in rheumatoid arthritis. Ann Rheum Dis 65: $746-752$

\section{Chondrocalcinosis does not worsen cartilage loss in knee OA}

Chondrocalcinosis (the deposition of calcium pyrophosphate dihydrate crystals in hyaline articular and/or meniscal fibrocartilage) is not associated with cartilage loss in osteoarthritic knees, say Neogi et al. Their finding is not new, but is important, as concerns about the sensitivity of radiographic detection of cartilage loss brought the findings of earlier, similar studies into question.

Neogi and colleagues used longitudinal MRI assessments to study 265 knees in 265 patients (mean age 67 years; $88 \%$ white; $42 \%$ female) with symptomatic osteoarthritis from the Boston Osteoarthritis Knee Study. Findings were then compared with those from a second cohort of 230 individuals (mean age 74 years; $69 \%$ female; $49 \%$ white) from the Health, Aging, and Body Composition Study, who had radiographic evidence of osteoarthritis.

Chondrocalcinosis was present in 23 of the 265 knees included in the Boston study, and was associated with a markedly lower risk of cartilage loss (two-fifths of that in knees without chondrocalcinosis). This reduced risk remained when just knees with damaged menisci were analyzed. A similar trend, perhaps suggesting a protective role of chondrocalcinosis, was observed in the 373 knees in the Health, Aging, and Body Composition study.

The authors suggest that chondrocalcinosis might indicate the presence of hypertrophic chondrocytes, which are associated with dysregulated matrix synthesis-a putative repair mechanism in injured joints. Further investigation of the effect of incident chondrocalcinosis on cartilage loss is required, as too few cases occurred in this study.

\footnotetext{
Original article Neogi T et al. (2006) Lack of association between chondrocalcinosis and increased risk of cartilage loss in knees with osteoarthritis: results of two prospective longitudinal magnetic resonance imaging studies. Arthritis Rheum 54: 1822-1828
}

\section{Can early treatment of RA reduce patients' risk of cardiovascular events?}

The increased likelihood of cardiovascular events in patients with rheumatoid arthritis $(\mathrm{RA})$ is not caused solely by 'classic' risk factors, but by an atherogenic lipid profile in early disease, a prospective, single-center Greek study has revealed.

The study included 58 patients ( 44 women) with $R A$ of $<1$ year duration, who had not previously 